\title{
Surveillance des comportements face au VIH/sida chez les hommes ayant des rapports sexuels avec des hommes - Suisse, 1992-2004
}

\author{
H. Balthasar, A. Jeannin, F. Dubois-Arber \\ Institut universitaire de médecine sociale et préventive, Lausanne, Suisse
}

Submitted: 4 July 2005; Revised: 24 March, 5 May 2006; Accepted: 2 June 2006

\begin{abstract}
Surveillance of HIV/AIDS - related behaviors among men who have sex with men - Switzerland, 1992-2004

Objective: To update (1992-2004, Switzerland) trends in behaviors and opinions relative to HIV prevention among men who have sex with men.

Methods: A cross sectional survey, repeated five times since 1992, among readers of the gay press and members of gay associations.

Results: An increase was observed in the median number of partners (1994: 5; 2004: 7, $\mathrm{p}<0.05$ ), in the proportion of participants who practiced anal penetration with their stable partner (1992: $71 \% ; 2004: 80 \%, p<0.05)$ or with casual partners (1994: $59 \%$ 2004: $73 \%$; $p<0.05$ ). Non systematic use of condoms with casual partners during anal penetration increased between 1994 and 2004 ( $9 \%$ to $20 \%$; p 0.05). Combination therapies gave rise to growing pessimism among participants regarding the decrease in preventive behaviors among homosexuals.

Conclusions: The slow trend towards a reduction of safer sex practices continues among men who have sex with men. It is necessary to strengthen prevention interventions in this population.
\end{abstract}

Keywords: Homosexuals - HIV prevention - Sexual behavior - Trends - Switzerland

En Suisse, ainsi que dans d'autres pays d'Europe occidentale, la première moitié de la décennie 2000 a été marquée par une recrudescence des nouveaux diagnostics d'infection à VIH, notamment parmi les hommes ayant des rapports sexuels avec des hommes (HSH) (Hamers \& Downs 2004). En Suisse, le nombre estimé de tests positifs rapportés à ce groupe de transmission a fortement augmenté entre 2001 et 2002 et s'est stabilisé en 2003 et 2004 (Office fédéral de la santé publique (OFSP) 2004).

La résurgence du VIH parmi les HSH s'explique principalement par la péjoration des comportements préventifs, dont les premiers signes ont été observés dans la seconde moitié des années 1990 (Moreau-Gruet et al. 2006), au moment où les multithérapies faisaient leur apparition.

Cet article a pour objectif d'actualiser, pour l'année 2004, les tendances relatives aux comportements et aux attitudes des HSH par rapport au VIH/sida, notamment dans le contexte actuel où les multithérapies sont disponibles. Les données présentées proviennent d'une enquête transversale (Swiss Gaysurvey), répétée sept fois depuis 1987, sur mandat de l'Office fédéral de la santé publique, dans le cadre de l'évaluation de la surveillance des comportements face au VIH/sida en Suisse (Dubois-Arber et al. 1999; Dubois-Arber et al. 2003).

\section{Méthodes}

L'enquête repose sur un échantillon de convenance. Les répondants ont été interrogés par le biais d'un questionnaire auto-administré, conçu en français, puis traduit en allemand et en italien.

La population visée par l'enquête est formée des HSH qui résident en Suisse. Les répondants n'ayant jamais eu de rapports avec un partenaire de même sexe et se définissant exclusivement comme hétérosexuels ont été exclus de l'échantillon.

Pour atteindre la population visée, les questionnaires ont été encartés dans les journaux d'audience nationale et régionale 
s'adressant spécifiquement à un lectorat homosexuel (4 à 6 titres selon les années). La grande majorité des associations homosexuelles actives sur le territoire ont également participé au recrutement en envoyant le questionnaire, par courrier, à leurs membres. En 2004, les saunas gays - identifiés sur la base de leur association faîtière - ont mis le questionnaire à disposition de leur clientèle. Enfin, lors de la dernière édition, le questionnaire pouvait également être rempli en ligne sur Internet. L'enquête y était annoncée grâce à des bannières publicitaires placées sur les sept sites gays suisses les plus fréquentés par des HSH.

Comme lors des éditions précédentes, à l'exception de l'enquête de 1992, la récolte des données a eu lieu durant les mois d'été (période durant laquelle les journaux ont souvent un tirage plus important). En 2004, dans le but de préserver la comparabilité de l'échantillon 'papier', l'enquête 'Internet' n'a commencé que début septembre. A ce moment, $86 \%$ de l'échantillon 'papier' était déjà constitué. Le questionnaire étant anonyme, il n'est pas possible d'identifier d'éventuels doublons.

Les questions portaient principalement sur les caractéristiques sociodémographiques des répondants et leurs comportements au cours des 12 mois précédant l'enquête, soit le nombre de partenaires, les pratiques sexuelles et l'utilisation de préservatifs avec les partenaires stables et occasionnels, ainsi que les modes de vie (lieux fréquentés, consommation de substances psychotropes). Les répondants étaient également interrogés sur la fréquence de leur recours au test VIH et sur leur statut sérologique. Dans la perspective d'une analyse de tendance, ces questions sont restées identiques depuis l'enquête de 1992. Dès 1997, des questions relatives aux connaissances et attitudes par rapport aux multithérapies ont été ajoutées et suivies.

Les comportements préventifs sont mesurés à l'aide de deux séries d'indicateurs. La première série mesure l'usage de préservatifs lors de la pénétration anale au cours des 12 derniers mois (réponses proposées: jamais, parfois, toujours) selon le type de partenaire (stable vs occasionnel). Cette distinction est nécessaire dès lors que l'usage de préservatifs est fortement conditionné par le type de relation entre les partenaires (Bochow et al. 1994) et que, dans le cadre d'une relation stable, il existe d'autres stratégies de protection que le seul usage de préservatifs (Moreau-Gruet et al. 2001). La seconde série ne contient qu'un indicateur et mesure le niveau général d'exposition au risque. Il est donné par la question suivante: «Ces 12 derniers mois, avez-vous pratiqué au moins une fois une pénétration anale non protégée avec un partenaire dont vous ne connaissiez pas le statut sérologique ou qui était d'un statut sérologique différent du vôtre?». $\mathrm{S}$ 'agissant des connaissance et des attitudes par rapport aux multithérapies, les répondants devaient indiquer, à l'aide d'une échelle à quatre positions, l'intensité de leur accord ou de leur désaccord avec trois énoncés relatifs, d'une part, à leurs propres attitudes et, d'autre part, à celles des homosexuels en général.

\section{Analyses}

Cet article présente essentiellement des analyses de tendance portant sur la période couverte par les cinq dernières enquêtes (1992, 1994, 1997, 2000, 2004). Les données ont fait l'objet d'analyses bivariées avec SPSS 12.0 (SPSS Inc. Chicago Il 60606-6307 USA) et STATA ${ }^{\text {TM }} 8.0$ (Statacorp LP, 4905 Lakeway Drive, TX 77845 USA). La signification statistique des tendances observées est évaluée à l'aide d'un test non paramétrique (nptrend) fourni par STATA ${ }^{\mathrm{TM}}$. Les proportions rapportées dans les tableaux sont accompagnées d'intervalles de confiance $(95 \%)$ calculés selon la méthode binomiale exacte. La signification statistique des comparaisons entre catégories à l'intérieur d'une même vague d'enquête a été mesurée avec le CHI-2 de Pearson.

Afin de préserver la comparabilité des données à travers le temps, il n'a pas été tenu compte des données de l'échantillon constitué sur Internet, dont la structure sociodémographique est jugée trop différente de celle de l'échantillon 'papier' (Balthasar et al. 2005).

Les données ont été stratifiées par âge (<30 ans vs. $\geq 30$ ans) en raison du vieillissement de la population répondante au fil des enquêtes. Afin de simplifier la présentation des résultats, les données de 2004 sont fréquemment comparées à celles de 1994, l'année d'enquête où les indicateurs d'exposition au risque étaient au niveau le plus bas.

\section{Participation en 2004}

Après exclusion des répondants non éligibles $(\mathrm{n}=15)$, l'échantillon de 2004 est composé de 1158 répondants, soit un effectif comparable aux éditions précédentes (Tableau 1). En 2004, 44\% des répondants ont rempli un questionnaire diffusé par une association, $42 \%$ ont complété un questionnaire encarté dans un journal, et $7 \%$ ont utilisé un questionnaire transmis par un établissement. La proportion de répondants établis en Suisse romande a fortement augmenté lors de la dernière enquête en raison d'une plus large diffusion du questionnaire: ceux-ci représentent environ $30 \%$ de l'échantillon en 2004, contre environ $20 \%$ en 1997 et 2000 (Tableau 1). 


\begin{tabular}{|c|c|c|c|c|c|}
\hline & 1992 & 1994 & 1997 & 2000 & 2004 \\
\hline Effectif & $N=934$ & $N=1195$ & $N=1097$ & $N=918$ & $N=1158$ \\
\hline Age médian (années) & 35 & 33 & 34 & 37 & 38 \\
\hline \multicolumn{6}{|l|}{ Classe d'âge } \\
\hline$<20$ ans & 0.4 & 1.2 & 1.3 & 1.2 & 1.6 \\
\hline $20-29$ ans & 30.5 & 33.4 & 24.2 & 16.3 & 18.3 \\
\hline$\geq 30$ ans & 68.7 & 65.4 & 74.1 & 81.9 & 80.0 \\
\hline Non réponse & 0.3 & & 0.4 & 0.5 & 0.1 \\
\hline \multicolumn{6}{|l|}{ Formation } \\
\hline Primaire/secondaire & 3.4 & 2.9 & 2.8 & 3.3 & 3.0 \\
\hline Formation sans maturité & 52.1 & 44.7 & 45.5 & 41.7 & 37.0 \\
\hline Formation avec maturité & 44.1 & 51.5 & 51.6 & 54.6 & 59.8 \\
\hline Non réponse & 0.3 & 0.8 & 0.1 & 0.4 & 0.2 \\
\hline \multicolumn{6}{|l|}{ Type de ménage } \\
\hline Seul & 60.3 & 51.2 & 56.5 & 55.1 & 53.6 \\
\hline En couple avec part. masc. & 20.1 & 25.5 & 25.1 & 28.4 & 26.6 \\
\hline Avec votre femme, amie & 5.1 & 3.5 & 2.6 & 3.5 & 2.3 \\
\hline Avec des amis & 9.1 & 11.8 & 9.6 & 7.4 & 11.6 \\
\hline Avec votre famille & 5.1 & 7.6 & 6.2 & 5.3 & 5.9 \\
\hline Non réponse & 0.2 & 0.3 & 0.1 & 0.2 & \\
\hline \multicolumn{6}{|l|}{ Zone de résidence } \\
\hline Commune rurale & 19.9 & 20.3 & 21.1 & 19.7 & 19.9 \\
\hline Ville de moins de 100000 habitants & 25.4 & 23.4 & 28.4 & 30.7 & 28.8 \\
\hline Ville de plus de 100000 habitants & 54.3 & 50.2 & 49.2 & 49.3 & 50.4 \\
\hline Non réponse & 0.4 & 6.1 & 1.3 & 0.2 & 0.9 \\
\hline \multicolumn{6}{|l|}{$\begin{array}{l}\text { Membre d'une association } \\
\text { homosexuelle }\end{array}$} \\
\hline Oui & 30.5 & 41.3 & 32.2 & 33.6 & 36.3 \\
\hline Non & 19.5 & 55.9 & 65.3 & 64.8 & 62.6 \\
\hline Non réponse & 50.0 & 2.8 & 2.6 & 1.6 & 1.1 \\
\hline \multicolumn{6}{|l|}{ Langue du questionnaire } \\
\hline Allemand & 77.3 & 77.7 & 74.4 & 75.3 & 67.4 \\
\hline Français & 22.7 & 22.3 & 25.6 & 21.8 & 31.3 \\
\hline Italien & & & & 2.9 & 1.4 \\
\hline \multicolumn{6}{|l|}{ Région linguistique } \\
\hline $\mathrm{CH}$ allemande & & & 64.6 & 54.6 & 64.8 \\
\hline $\mathrm{CH}$ romande & & & 22.7 & 17.8 & 28.7 \\
\hline $\mathrm{CH}$ italienne & & & 0.5 & 1.6 & 1.6 \\
\hline Non réponse & & & 12.2 & 26.0 & 4.9 \\
\hline
\end{tabular}

Tableau 1 Caractéristiques sociodémographiques des répondants, par vague d'enquête (Suisse 1992, 1994, 1997 2000, 2004)

\section{Résultats}

\section{Caractéristiques sociodémographiques}

On observe un vieillissement important de la population répondante au fil des enquêtes. L'âge médian est passé de 33 à 38 ans entre 1994 et 2004 (Tableau 1). Le niveau de formation - vraisemblablement plus élevé que dans la population générale (Moreau-Gruet \& Dubois-Arber 1995) - a également augmenté. En 2004, près de $60 \%$ des répondants ont une maturité et plus d'un tiers a suivi une filière universitaire. Les autres caractéristiques sociodémographiques sont restées relativement stables. Il s'agit d'une population majoritairement urbaine: plus d'un répondant sur deux vit dans une ville de plus de 100000 habitants, plus de la moitié vit seul et un répondant sur quatre partage un logement avec un partenaire masculin. Les membres d'associations homosexuelles repré- sentent entre $30 \%$ et $40 \%$ de l'échantillon selon les vagues d'enquête.

\section{Recours au test de dépistage et statut sérologique}

En 1992, 72\% des répondants avaient recouru au moins une fois dans la vie au test VIH (Tab. 2). Cette proportion a lentement augmenté au fil des enquêtes pour atteindre $83 \%$ en $2004(\mathrm{p}<0.001)$. Parmi les répondants de moins de 30 ans, la proportion de personnes testées au cours de la vie est restée stable (environ 70\%) alors qu'elle a augmenté chez leurs aînés passant de $71 \%$ en 1992 à $86 \%$ en 2004 $(\mathrm{p}<0.001)$.

En 2004, parmi les répondants testés, $44 \%$ l'ont été au cours des 12 mois précédant l'enquête. Le pourcentage de séropositifs (Tab. 2) est resté stable parmi les répondants testés entre 1992 et 2004 (environ 10\%). La prévalence du VIH est moins 
élevée parmi les répondants de moins de 30 ans que parmi leurs aînés (2004: 3 \% vs. $10 \%$; p 0.001).

\section{Activité sexuelle et modes de vie}

A l'exception du nombre de partenaires, les caractéristiques de l'activité sexuelle des répondants ont peu évolué avec les années (Tab. 2). Environ $90 \%$ définissent leur orientation comme exclusivement homosexuelle (1992: 88\%; 2004: $93 \% ; \mathrm{p}<0.001)$. La proportion de répondants ayant eu une orientation bisexuelle a diminué au cours du temps, passant de $11 \%$ en 1992 à $4 \%$ en 2004 (p < 0.001).

La proportion de répondants déclarant au moins une relation stable au cours des 12 derniers mois a fortement augmenté entre 1992 et 1994, puis est restée stable. En 2004, environ deux tiers des répondants ont eu un partenaire stable. Les relations non exclusives sont fréquentes: c'est le cas d'un tiers des relations stables déclarées par les répondants de moins de 30 ans et de la moitié de celles mentionnées par leurs aînés.

Un peu moins de $80 \%$ des répondants, lors de chaque vague d'enquête, déclarent avoir eu des partenaires occasionnels au cours des 12 derniers mois. Ce mode de relation est légèrement moins fréquent parmi les répondants de moins de 30 ans (2004: 70\%) que chez leurs aînés (2004: $78 \%$ ).

Le nombre de partenaires sexuels au cours des 12 derniers mois a sensiblement augmenté à partir de la seconde moitié des années 1990 (Tab. 2). Entre 1994 et 2004, la médiane est passée de 5 à 7 ( $\mathrm{p}<0.05)$. L'augmentation est particulièrement marquée chez les plus de 30 ans (1994: 6; 2004: 8; p $<0.05)$, tandis que la médiane est restée relativement stable parmi les plus jeunes (5 partenaires). Entre 2000 et 2004, on note également, parmi les plus de 30 ans, une augmentation du nombre médian de partenaires avec lesquels les répondants ont pratiqué la pénétration anale (2000: 1 partenaire; 2004: 2 partenaires; $\mathrm{p}<0.001)$.

La fréquentation d'espaces conçus ou reconnus pour favoriser des rencontres sexuelles (saunas, lieux extérieurs de drague) a sensiblement évolué depuis 1997, en particulier parmi les répondants de moins de 30 ans. Un répondant sur deux a fréquenté régulièrement ou occasionnellement des saunas gays. Leur fréquentation a sensiblement baissé parmi les répondants de moins de 30 ans $^{1}$ (1997: 41\%; 2004: $27 \%$; p < 0.05) alors qu'elle est restée stable chez leurs aînés (environ $55 \%$ ). La fréquentation régulière ou occasionnelle des lieux extérieurs de drague (parcs, WC publics, plages) a également baissé, passant de $64 \%$ en 1997 à $55 \%$ en 2004 (p < 0.05). Cette tendance est à nouveau plus marquée parmi les moins de 30 ans (1997: $57 \%$; 2004: $38 \%$, p < 0.001) que chez leurs aînés

\footnotetext{
${ }^{1}$ L'accès des saunas étant interdit aux mineurs, les répondants de moins de 18 ans n'ont pas été considérés dans le calcul de ce pourcentage.
}

(1997: 66\%; 2004: 59\%; p 0.05). La recherche de partenaires via Internet a, quant à elle, connu un véritable essor, quel que soit l'âge des répondants: en 2004, $42 \%$ des répondants y ont eu recours, contre $25 \%$ en $2000(\mathrm{p}<0.05)$.

\section{Exposition au risque}

La pratique de la pénétration anale ainsi que l'utilisation non systématique de préservatifs dans cette circonstance (exposition au risque) suivent une tendance ascendante depuis l'enquête de 1997. Cette évolution est particulièrement perceptible dans le contexte de relations occasionnelles (Tab. 3). Entre 1994 (année d'enquête où les indicateurs de risque sont au plus bas) et 2004, la proportion de répondants ayant pratiqué la pénétration anale avec ces partenaires est passée de $56 \%$ à $73 \%(\mathrm{p}<0.001)$. La proportion de répondants n'ayant pas systématiquement utilisé de préservatifs lors de la pénétration anale avec des partenaires occasionnels est, quant à elle, passée de $9 \%$ en 1994 à $20 \%$ en 2004 (p<0.001): de $7 \%$ à $17 \%$ chez les moins de 30 ans $(\mathrm{p}<0.01)$ et de $10 \%$ à $21 \% \mathrm{chez}$ leurs aînés $(\mathrm{p}<0.001)$.

Avec le partenaire stable, compte tenu des stratégies de protection alternatives (par exemple: fidélité mutuelle exclusive), l'utilisation de préservatifs est moins élevée qu'avec les partenaires occasionnels (Tab. 3). Hormis une augmentation de la pratique de la pénétration anale (1994: $73 \%$; 2004: 80\%; p $<0.05$ ), on n'observe pas de changements en ce qui concerne l'utilisation du préservatif dans cette circonstance.

Cette observation appelle toutefois une analyse plus circonstanciée, qui tienne compte du statut sérologique des deux partenaires: couples séroconcordants négatifs et positifs, couples sérodifférents et couples indéterminés (Tab. 4). En 2004, $51 \%$ de couples sont séroconcordants négatifs (1994: $40 \%$ ) et $40 \%$ sont indéterminés (1994: $51 \%$ ). Les couples séroconcordants positifs et les couples sérodifférents représentent ensemble moins de $10 \%$ des relations stables. Lorsque le statut sérologique des deux partenaires est connu, la mention de rapports non protégés est d'autant plus faible que le risque de transmission est manifeste (couples sérodiscordants). Dans les couples indéterminés, la mention de rapports non protégés est particulièrement élevée (33\%).

\section{Niveau général d'exposition au risque}

L'estimation globale du niveau d'exposition au risque au sein de la population répondante est donnée par la proportion de répondants ayant eu au moins une pénétration anale non protégée avec un partenaire de statut sérologique différent ou inconnu au cours des 12 mois précédant l'enquête (indépendamment des pratiques sexuelles et du type de partenaire). La valeur de cet indicateur, après avoir diminué entre 1992 (16.5\%) et $1994(10.7 \%)$, a augmenté en 2000 (14.2\%), puis 


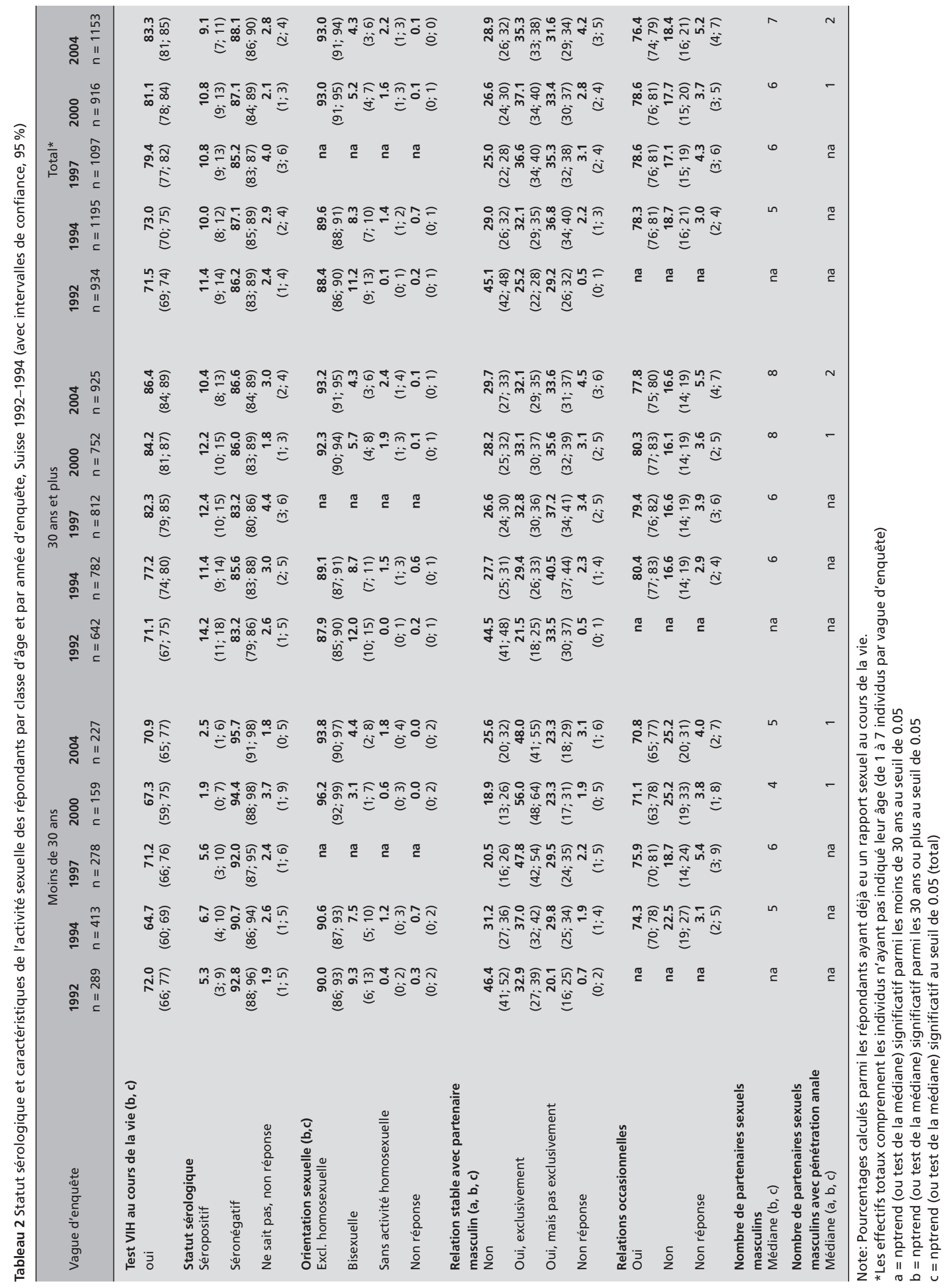




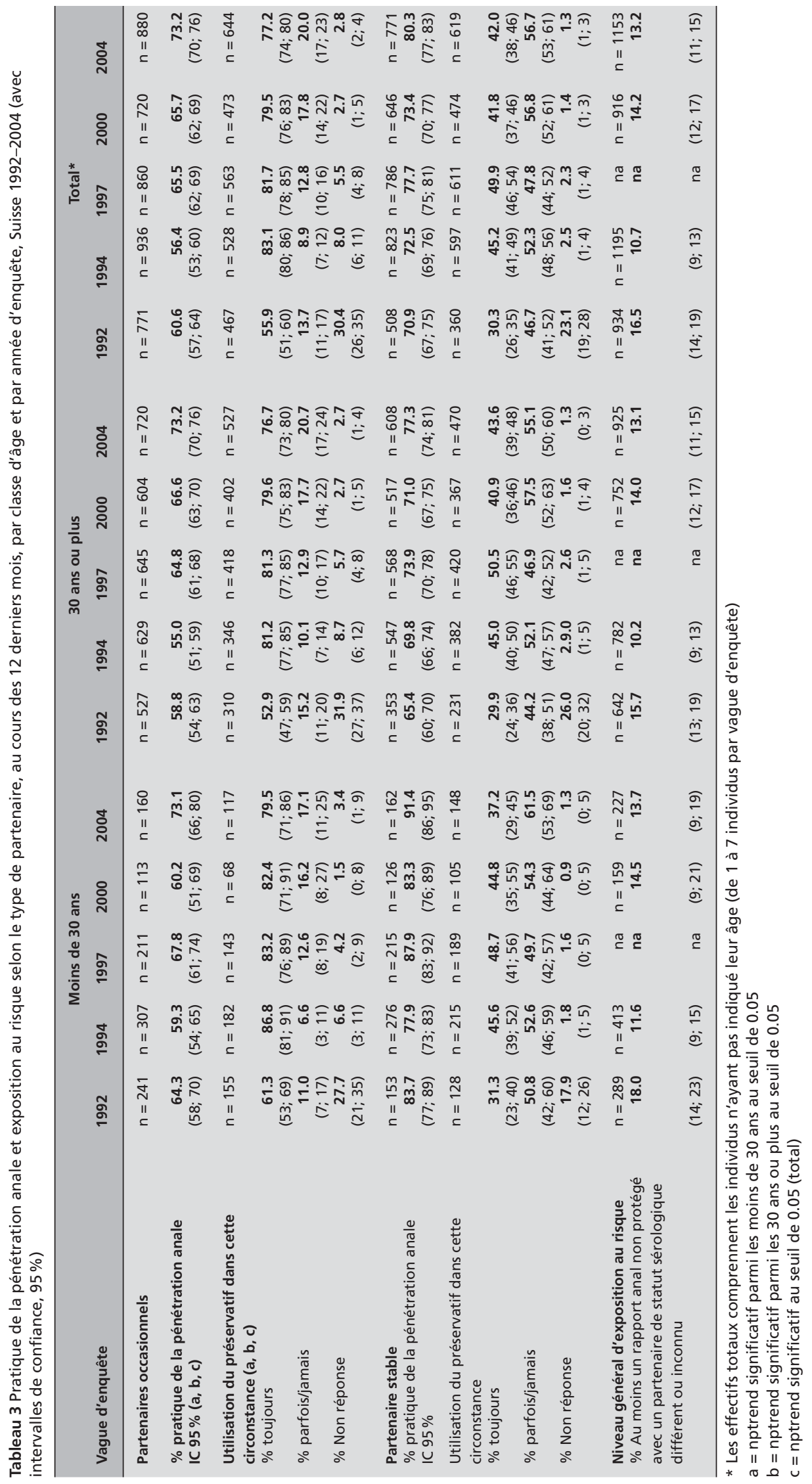


Tableau 4 Type de relations stables ${ }^{\text {a }}$ selon le statut sérologique des deux partenaires et proportion des répondants déclarant des rapports non protégés avec le partenaire stable dans chaque type de couple; par année d'enquête, Suisse 1994-2004 (avec intervalles de confiance, 95\%)

\begin{tabular}{|c|c|c|c|c|c|}
\hline Types de relations stables & $\%$ & $\begin{array}{l}1994 \\
n=823\end{array}$ & $\begin{array}{l}1997 \\
n=786\end{array}$ & $\begin{array}{l}2000 \\
n=646\end{array}$ & $\begin{array}{l}2004 \\
n=771\end{array}$ \\
\hline \multirow[t]{2}{*}{$\begin{array}{l}\text { Séroconcordants négatifs: } \\
\text { les deux partenaires séronégatifs }\end{array}$} & $\%$ des relations stables & $\begin{array}{r}39.9 \\
(36 ; 43)\end{array}$ & $\begin{array}{r}42.0 \\
(39 ; 46)\end{array}$ & $\begin{array}{r}48.1 \\
(44 ; 52)\end{array}$ & $\begin{array}{r}50.7 \\
(47 ; 54)\end{array}$ \\
\hline & dont \% mentionnant des rapports non protégés & $\begin{array}{r}55.2 \\
(50 ; 61)\end{array}$ & $\begin{array}{r}55.2 \\
(50 ; 61)\end{array}$ & $\begin{array}{r}61.0 \\
(55 ; 67)\end{array}$ & $\begin{array}{r}61.6 \\
(56 ; 66)\end{array}$ \\
\hline \multirow[t]{2}{*}{$\begin{array}{l}\text { Séroconcordants positifs: } \\
\text { les deux partenaires séropositifs }\end{array}$} & $\%$ des relations stables & $\begin{array}{r}2.1 \\
(1 ; 3)\end{array}$ & $\begin{array}{r}2.2 \\
(1 ; 3)\end{array}$ & $\begin{array}{r}1.4 \\
(1 ; 3)\end{array}$ & $\begin{array}{r}1.7 \\
(1 ; 3)\end{array}$ \\
\hline & dont \% mentionnant des rapports non protégés & $\begin{array}{r}35.3 \\
(14 ; 62)\end{array}$ & $\begin{array}{r}29.4 \\
(10 ; 56)\end{array}$ & $\begin{array}{r}55.6 \\
(21 ; 86)\end{array}$ & $\begin{array}{r}69.2 \\
(39 ; 91)\end{array}$ \\
\hline \multirow[t]{2}{*}{$\begin{array}{l}\text { Sérodifférents: un partenaire } \\
\text { séropositif, l'autre séronégatif }\end{array}$} & $\%$ des relations stables & $\begin{array}{r}7.0 \\
(5 ; 9)\end{array}$ & $\begin{array}{r}8.3 \\
(6 ; 10)\end{array}$ & $\begin{array}{r}9.6 \\
(7 ; 12)\end{array}$ & $\begin{array}{r}7.5 \\
(6 ; 10)\end{array}$ \\
\hline & dont \% mentionnant des rapports non protégés & $\begin{array}{r}7.1 \\
(2 ; 17)\end{array}$ & $\begin{array}{r}7.7 \\
(3 ; 17)\end{array}$ & $\begin{array}{r}13.1 \\
(6 ; 24)\end{array}$ & $\begin{array}{r}8.8 \\
(3 ; 19)\end{array}$ \\
\hline \multirow{2}{*}{$\begin{array}{l}\text { Indéterminés: un partenaire ou } \\
\text { les deux de statut sérologique } \\
\text { inconnu }\end{array}$} & $\%$ des relations stables & $\begin{array}{r}51.0 \\
(48 ; 54)\end{array}$ & $\begin{array}{r}47.6 \\
(44 ; 51)\end{array}$ & $\begin{array}{r}40.9 \\
(37 ; 45)\end{array}$ & $\begin{array}{r}40.1 \\
(37 ; 44)\end{array}$ \\
\hline & dont \% mentionnant des rapports non protégés & $\begin{array}{r}32.6 \\
(28 ; 37)\end{array}$ & $\begin{array}{r}28.0 \\
(23 ; 33)\end{array}$ & $\begin{array}{r}27.2 \\
(22 ; 33)\end{array}$ & $\begin{array}{r}33.3 \\
(28 ; 39)\end{array}$ \\
\hline
\end{tabular}

a Relations stables au cours des 12 derniers mois telles que déclarées par les répondants

b Il n'y a pas de données pour 1992 car les répondants n'étaient pas interrogés sur le statut sérologique de leur partenaire.

Note: nptrends non significatifs au seuil de 0.05

est resté stable en 2004 (13\%). Entre 1992 et 2004, aucune tendance significative n'est observée à partir de cet indicateur. C'est parmi les répondants séropositifs qu'il atteint ses valeurs les plus hautes (2004: $23 \%$ ), alors qu'il est proche de la moyenne dans les autres groupes sérologiques (séronégatifs: $13 \%$; non testés: $11 \%$ ). Ces différences ne sont toutefois pas statistiquement significatives.

\section{Connaissances et attitudes envers les multithérapies}

Depuis 1997, les connaissances des répondants par rapport aux multithérapies se sont sensiblement améliorées. En 2004, $5 \%$ des répondants déclarent ne pas en avoir entendu parler, contre $23 \%$ en 1997 ( $\mathrm{p}<0.001$ ). En 2004, parmi les personnes ayant entendu parler de ces traitements, plus d'un tiers estiment ne pas être bien informées. Les croyances erronées par rapport à l'efficacité des traitements, comme la guérison définitive ou l'arrêt de la transmission du virus, sont devenues insignifiantes en 2004.

Les opinions relatives à l'impact des multithérapies sur les comportements préventifs sont contrastées. Un écart substantiel se creuse entre les attitudes attribuées à autrui et celles déclarées pour soi-même (Figure 1). Ainsi, entre 1997 et 2004, la proportion de répondants qui adhèrent à l'idée selon laquelle, «avec les multithérapies, les homosexuels font moins attention à la prévention», est passée de $32 \%$ à $71 \%(\mathrm{p}<0.001)$. La proportion de ceux qui se reconnaissent personnellement dans cet énoncé a également fortement augmenté (1997: $5 \%$; 2004: $10 \%$; p < 0.001), mais ne concerne cependant qu'une minorité de répondants (Fig. 1.a). Une proportion croissante de répondants (1997: 29\%; 2004: 62\%; p < 0.001) estime que la peur de devenir séropositif diminue parmi les homosexuels en général, alors que cette proportion a diminué au niveau individuel (1997: 15\%; 2004: $11 \%$; p < 0.05) (Fig. 1.b). On observe enfin une augmentation de la proportion de répondants estimant que, en raison des multithérapies, les homosexuels choisissent de prendre des risques (2000: 57\%; 2004: 69\%, p < 0.001). En revanche, une minorité d'individus, restée stable, s'attribue personnellement cette disposition (2000: 5\%; 2004: 7\%; n.s.) (Fig. 1.c).

\section{Discussion}

\section{Les limites de l'enquête}

Compte tenu de la difficulté à recruter un nombre suffisant de HSH par des enquêtes représentatives de la population générale, la plupart des études menées dans ce segment de population reposent sur des échantillons de convenance (Bochow et al. 1994; Elford et al. 2004a). On estime que les répondants, en tant que lecteurs de la presse communautaire ou membres d'associations homosexuelles, appartiennent au segment de la population homosexuelle le plus affirmé du point de vue des préférences sexuelles, le plus informé, voire le plus motivé en ce qui concerne la prévention du VIH. Ces biais de sélection conduisent probablement à surestimer le niveau de protection au sein de la population HSH. Cependant, dans la mesure où 


\section{Figure 1.a}

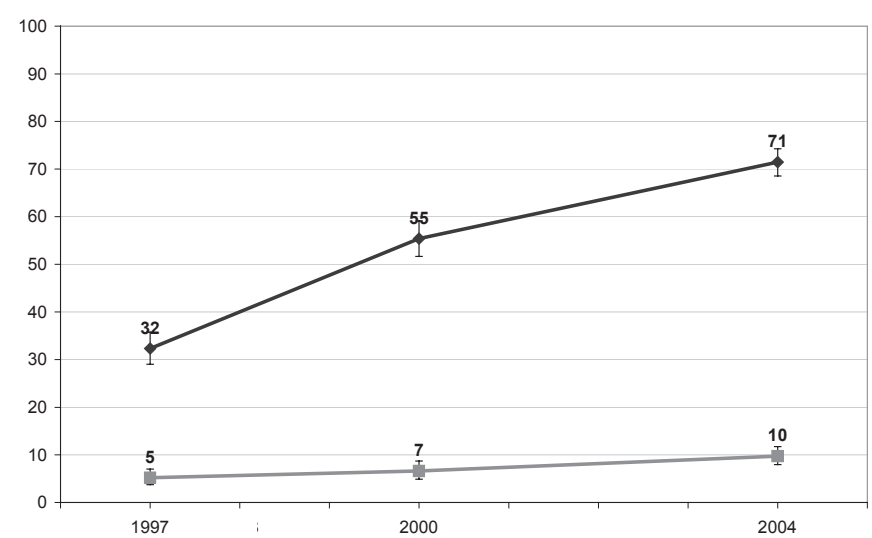

Figure 1.b

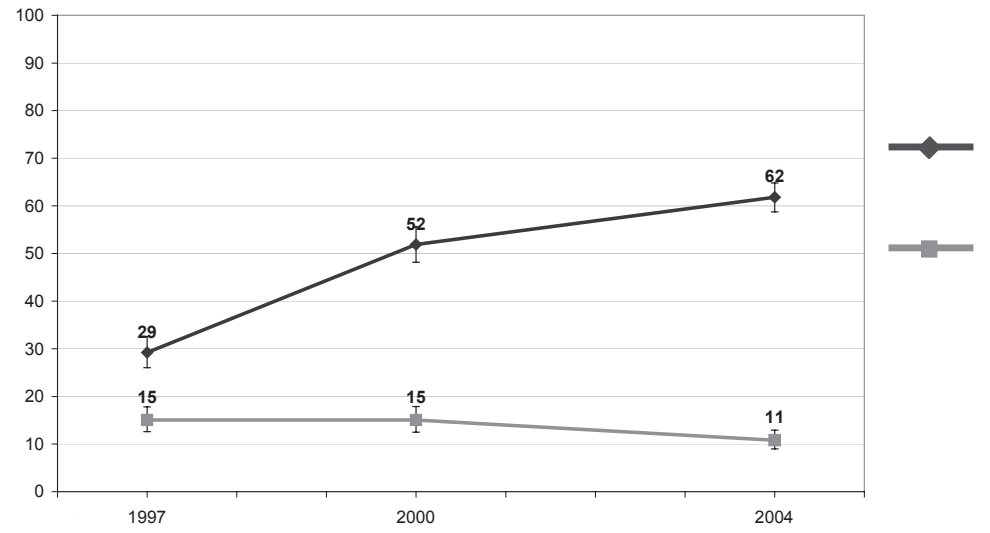

Figure 1.c

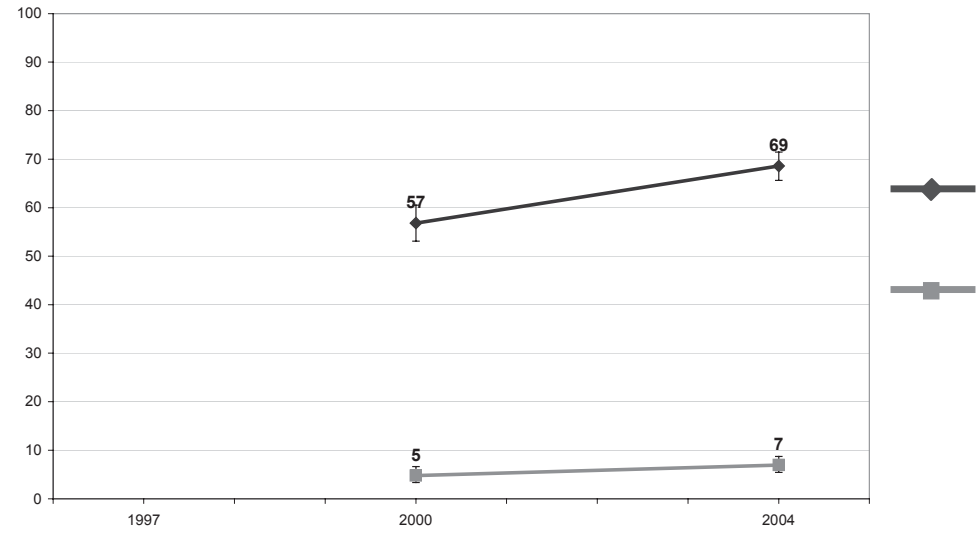

Les homosexuels font moins attention à la prévention du VIH $(p<0.001)$

Moi-même, je fais moins attention à la prévention du VIH $(p<0.05)$
Les homosexuels ont moins peur d'être ou de devenir séropositifs $(p<0.001)$

Moi-même, j'ai moins peur d'être ou de devenir séropositif $(p<0.001)$

De plus en plus les homosexuels font le choix de prendre des risques $(p<0.001)$

Moi-même, de plus en plus je fais le choix de prendre des risques (ns)

Figure 1 Proportion de répondants se déclarant «tout à fait d'accord» ou "d'accord» avec les énoncés suivants en lien avec les multithérapies, par année d'enquête, Suisse 1997-2004 (avec intervalles de confiance, $95 \%$ ) 
Surveillance des comportements face au VIH/sida chez les hommes ayant des rapports sexuels avec des hommes - Suisse, 1992-2004
Int J Public Health 52 (2007) 27-38

(c) Birkhäuser Verlag, Basel, 2007 ces biais sont restés relativement stables, on peut admettre que les tendances temporelles sont valides.

Les jeunes sont en général peu représentés dans les échantillons constitués à partir de questionnaires insérés dans la presse homosexuelle ou diffusés par les associations. D'une part, ces canaux de transmission ont une couverture limitée et, de ce fait, ne sont pas forcément accessibles aux plus jeunes. D'autre part, aussi bien l'acquisition d'une revue que l'adhésion à une association homosexuelle ne sont pas des démarches allant de soi car elles supposent, au préalable, le développement d'un certain sens identitaire (Balthasar 2004; Schiltz 1997).

Bien qu'il n'ait pas été envisagé, pour des raisons méthodologiques, de tenir compte, dans l'analyse des tendances, des données récoltées en 2004 sur Internet, il est important de souligner ici que l'utilisation de cette technologie a permis d'atteindre des publics peu touchés par le questionnaire imprimé. Les expériences réalisées en Suisse (Balthasar et al. 2005), comme dans d'autres pays (Bochow et al. 2004b; Elford et al. 2004b; Ross et al. 2000), montrent que les répondants recrutés sur Internet sont sensiblement plus jeunes, ont un niveau de formation moins élevé et vivent davantage dans des régions périphériques. Il est d'ores et déjà prévu d'intégrer ce mode de recrutement des répondants aux futures enquêtes de surveillance des comportements.

\section{Augmentation des expositions au risque}

Les données recueillies en 2004 s'inscrivent dans le prolongement des tendances observées dès la seconde moitié des années 1990. Premièrement, entre 1994 et 2004, on observe une augmentation importante de la pratique de la pénétration anale, aussi bien dans le cadre de relations stables que lors de relations occasionnelles. Deuxièmement, le nombre de partenaires sexuels augmente sensiblement, en particulier parmi les répondants de 30 ans ou plus. L'augmentation du nombre de partenaires, avec lesquels les répondants ont pratiqué la pénétration anale, confirme la progression de cette pratique au sein de la population HSH. Enfin, l'utilisation de préservatifs lors de la pénétration anale avec des partenaires occasionnels régresse. Confirmée en 2000 parmi les répondants de 30 ans ou plus (Moreau-Gruet et al. 2006), cette tendance s'est généralisée en 2004 à l'ensemble de l'échantillon.

Des tendances similaires ont été identifiées dans d'autres pays d'Europe occidentale ainsi qu'en Amérique du Nord. Si la variété des procédures d'échantillonnage hypothèque la comparaison des indicateurs entre les pays, les tendances mises au jour convergent. En l'occurrence, une diminution régulière de la protection dans le contexte de relations occasionnelles a été observée en France (période: 1991-2000) (Bochow et al. 2004a), en Allemagne (1991-2003) (Bochow et al. 2004b), au Royaume-Uni (1998-2003) (Elford et al. 2004a), en Australie (1999-2003) (Van de Ven et al. 2004) et en Californie (1994-2001) (Chen et al. 2002). La situation est, en revanche, restée stable en Espagne (1995-2002) (Casabona et al. 2003). Les indicateurs relatifs au nombre annuel de partenaires sexuels et à la pratique de la pénétration anale suivent, comme en Suisse, une tendance ascendante en France (Bochow et al. 2004a) et en Allemagne (Bochow et al. 2004b). Il est possible que l'augmentation du nombre de partenaires soit liée au développement d'Internet (forums de discussion, messageries instantanées), soit une ressource de plus en plus utilisée par les répondants dans leur recherche de partenaires. De fait, cet outil facilite considérablement les rencontres étant donné la gratuité des services concernés, l'affranchissement des contraintes géographiques, et la possibilité pour les utilisateurs d'expliciter leurs préférences.

S'agissant des comportements préventifs entre partenaires d'une relation stable, la situation observée en Suisse ne s'est guère améliorée. Sous réserve de l'application de stratégies de protection alternatives et négociées entre les deux partenaires (Moreau-Gruet et al. 2001), c'est dans les couples séroconcordants (positifs ou négatifs) que le préservatif est le moins utilisé. Dans les couples dont le statut sérologique de l'un ou des deux partenaires est indéterminé, le taux d'exposition au risque reste particulièrement élevé. Or, ces couples représentent, en 2004, près de la moitié des relations stables.

Malgré le relâchement des comportements préventifs, la proportion de répondants déclarant avoir eu au moins un rapport non protégé avec un partenaire de statut sérologique différent ou inconnu, est restée stable en 2004 (13\%), après avoir augmenté entre 1994 et 2000 (de $9 \%$ à 14\%). La stabilité de cet indicateur est surprenante alors que le nombre de partenaires augmente et que la protection diminue dans le cadre de relations occasionnelles. Elle suggère que l'augmentation des rapports non protégés (avec des partenaires occasionnels) survient dans des situations qui demeureraient à faibles risques (partenaire connu, statut sérologique divulgué) ou qui seraient perçues comme telles par les répondants.

\section{Un pessimisme croissant à l'égard de la prévention}

La diffusion des multithérapies n'a pas suscité d'optimisme immodéré parmi les répondants. Au contraire, les enquêtes menées en Suisse et à l'étranger montrent que la grande majorité des HSH sont réalistes quant à l'efficacité des traitements (pas de guérison définitive), à la pénibilité des effets secondaires et/ou à la diminution de l'infectiosité des personnes séropositives (Elford et al. 2002; International Collaboration on HIV Optimism 2003; Knox et al. 2001; Stolte et al. 2004; Van de Ven et al. 2002). 
Cette réserve à l'égard des multithérapies n'a néanmoins pas empêché l'émergence, aussi bien dans les milieux de la prévention que parmi les $\mathrm{HSH}$, d'un certain pessimisme quant à l'évolution des comportements préventifs en relation avec le contexte thérapeutique actuel (Lert 2000). En Suisse, depuis 1997, cette attitude est montée en puissance parmi les répondants, si bien que, en 2004, une forte majorité pense que les homosexuels sont prêts à prendre davantage de risques à cause de l'existence des multithérapies. Cette opinion est cependant contredite par les comportements rapportés au niveau individuel: seule une minorité de répondants, par ailleurs stable, admet prendre davantage de risques en raison des possibilités thérapeutiques actuelles.

Concernant l'impact des multithérapies sur les comportements préventifs, la littérature n'est pas unanime. Certaines études ont établi une association entre une attitude 'optimiste' à l'égard des traitements et la prise de risque (International Collaboration on HIV Optimism 2003; Van de Ven et al. 2002), tandis que d'autres études ont infirmé cette hypothèse (Elford et al. 2002; Williamson \& Hart 2004). A notre connaissance, seule une étude de cohorte, réalisée à Amsterdam, est parvenue à démontrer qu'il existe un lien de causalité entre les opinions relatives aux multithérapies et l'engagement dans des pratiques à risque (Stolte et al. 2004).

\section{Zusammenfassung}

Beobachtung des HIV/AIDS-relevanten Verhaltens bei Männern mit sexuellen Beziehungen zu Männern - Schweiz 1992-2004

Ziel: Die Trends bei den Verhaltensweisen und Einstellungen bezüglich der HIV-Prävention bei Männern mit sexuellen Beziehungen zu Männern sollen aktualisiert werden.

Methoden: Die Datenbasis bildet ein Querschnittsurvey mit fünfmaliger Wiederholung seit 1992 bei Lesern von Presseerzeugnissen, die für ein homosexuelles Publikum bestimmt sind und bei Mitgliedern von Vereinen und Organisationen von Homosexuellen in der Schweiz.

Ergebnisse: Ein Anstieg wurde beobachtet beim Median der Anzahl Partner (1994: 5, 2004: 7, p < 0.05), beim Anteil der Teil-

\section{Conclusion}

Depuis la seconde moitié des années 1990, on observe une augmentation sensible de plusieurs facteurs de risque (nombre de partenaires et pratique de la pénétration anale), ainsi que des expositions au risque (rapports non protégés). Bien que la majorité des HSH interrogés continue de se protéger efficacement contre le VIH et que l'augmentation des expositions au risque soit de faible ampleur d'une enquête à l'autre, cette évolution n'en est pas moins régulière. Plus de 20 ans après le début de l'épidémie, à l'heure où le sida se conçoit de plus en plus comme une maladie chronique, la diminution progressive des comportements préventifs est source d'inquiétudes.

Il est nécessaire de renforcer les mesures de prévention et de poursuivre la surveillance des comportements relatifs au VIH/ sida parmi les HSH. Une prochaine enquête est d'ores et déjà programmée pour 2007.

\section{Remerciements}

Nous remercions chaleureusement toutes les personnes qui ont répondu à l'enquête, ainsi que les associations, les établissements, les sites Internet et les revues qui ont diffusé le questionnaire.

Aucun conflit d'intérêt n'est à signaler.

Cette étude a été financée par l'Office fédéral de la santé publique, Berne - Contrat 00.001343 nehmer, die anale Penetration praktizierten mit ihren festen Partnern (1994: $71 \%$; 2004: $80 \%, p<0.05)$ oder mit gelegentlichen Partnern (1994: $59 \%, 2004: 73 \%, p<0.05)$. Der nichtsystematische Gebrauch von Kondomen mit gelegentlichen Partnern bei analer Penetration stieg von 1994 bis 2004 an (von $9 \%$ auf $20 \%, p<0.05$ ). Die Kombinationstherapien gaben bei den Befragten Anlass zu steigendem Pessimismus bezüglich des präventiven Verhaltens bei Homosexuellen.

Schlussfolgerung: Der langsame Trend zu einer Reduktion von Safer Sex-Praktiken hält bei Männern mit sexuellen Beziehungen zu Männern an. Eine Stärkung der Präventionsinterventionen bei dieser Population ist notwendig. 


\section{Résumé}

Objectifs: Actualiser (1992-2004, en Suisse) les tendances relatives aux comportements et attitudes face au $\mathrm{VIH} /$ sida chez les hommes ayant des rapports sexuels avec des hommes (HSH).

Méthodes: Une enquête transversale par questionnaire, répétée à cinq reprises depuis 1992, auprès des membres d'associations homosexuelles et des lecteurs de la presse homosexuelle. Résultats: Le nombre médian de partenaires, au cours des 12 derniers mois, a augmenté (1994: 5; 2004: 7, p < 0.05), ainsi que la proportion de répondants pratiquant la pénétration anale avec leur partenaire stable (1992: $71 \%$; 2004: $80 \%$, p < 0.05) ou occasionnels (1994: $59 \%$; 2004: $73 \%$; $p<0.05$ ). L'utilisation non systématique de préservatifs lors de la pénétration anale avec les partenaires occasionnels a augmenté entre 1994 et 2004 (1994: $9 \%$; 2004: $20 \% ; p<0.05$ ). Les multithérapies suscitent parmi les répondants un pessimisme croissant quant à la diminution des comportements préventifs dans la population homosexuelle: les répondants tendent à surestimer cette évolution.

Conclusion: La lente diminution des comportements préventifs se poursuit parmi les HSH. Il faut renforcer les mesures de prévention dans ce segment de population.

\section{References}

Balthasar H (2004). Populations particulières pour la prévention du sida, les jeunes homosexuels et bisexuels masculins: revue de littérature. Lausanne: Institut universitaire de médecine sociale et préventive (Raisons de santé, 99).

Balthasar H, Jeannin A, Dubois-Arber F (2005). Augmentation des expositions au risque d'infection par le VIH chez les hommes ayant des rapports sexuels avec des hommes: premiers résultats de GAYSURVEY 04. Bulletin OFSP 48: 891-5.

Bochow M, Chiarotti F, Davies P et al (1994). Sexual behaviour of gay and bisexual men in eight European countries. AIDS Care 6(5): 533-49.

Bochow M, Jauffret M, Michel A, Schiltz, M-A. (2004a.) Les évolutions des comportements sexuels et les modes de vie à travers les enquêtes réalisées dans la presse gaie en France (1985-2000). In: Broqua C,. Lert F, Souteyrand Y, eds. Homosexualités au temps du sida: tension sociales et identitaires. Paris: Agence nationale de recherche sur le sida, 35-54.

Bochow M, Wright MT, Lange M (2004b). Schwule Männer und Aids: Risikomanagement in Zeiten der sozialen Normalisierung einer Infektionskrankheit [Eine Befragung im Auftrag der Bundeszentrale für gesundheitliche Aufklärung, Köln]. Berlin: Deutsche Aids-Hilfe e.V.

Casabona J et al. (2003). SIVES 2003 Annual report CEESCAT: intergrated HIV/AIDS surveillance system of Catalonia.Generalitat de Catalunya, Departament de Sanitat i Seguretat Social.
Chen SY, Gibson S, Katz, MH et al (2002). Continuing increases in sexual risk behavior and sexually transmitted diseases among men who have sex with men: San Francisco, Calif, 1999-2001, USA. Am J Public Health 92(9): 1387-8.

Dubois-Arber F, Jeannin A, Meystre-Agustoni $G$ et al. (2003). Evaluation de la stratégie de prévention du VIH/sida en Suisse: septième rapport de synthèse 1999-2003. Lausanne: Institut universitaire de médecine sociale et préventive (Raisons de santé, 90a).

Dubois-Arber F, Jeannin A, Spencer B (1999). Long term global evaluation of a national AIDS prevention strategy: the case of Switzerland. AIDS 13: 2571-82.

Elford J, Bolding G, Davis $M$, Sherr L, Hart $G$ (2004a). Trends in sexual behaviour among London homosexual men 1998-2003: implications for HIV prevention and sexual health promotion. Sex Transm Infect 80(6): 451-4.

Elford J, Bolding G, Davis M, Sherr L, Hart $G$ (2004b). Web-based behavioral surveillance among men who have sex with men: a comparison of online and offline samples in London, UK. J Acquir Immune Defic Syndr 35(4): 421-6.

Elford J, Bolding G, Sherr L (2002). High-risk sexual behaviour increases among London gay men between 1998 and 2001: what is the role of HIV optimism? AIDS 16(11): 1537-44.

Hamers FF, Downs AM (2004). The changing face of the HIV epidemic in western Europe: what are the implications for public health policies? Lancet 364(9428): 83-94.

International Collaboration on HIV Optimism (2003). HIV treatments optimism among gay men: an international perspective. J Acquir Immune Defic Syndr 32(5): 545-50.
Knox S, Van de Veen, Prestage G, Crawford J, Grulich A, Kippax S (2001). Increasing realism among gay men in Sydney about HIV treatments: changes in attitudes over time. Int J STD AIDS 12(5): 310-4.

Lert F (2000). Advances in HIV treatment and prevention: should treatment optimism lead to prevention pessimism? AIDS Care 12(6): $745-55$.

Moreau-Gruet F, Dubois-Arber F (1995). Les hommes aimant d'autres hommes: étude 1994. Lausanne: Institut universitaire de médecine sociale et préventive. (Cah Rech Doc IUMSP, no. 120.5).

Moreau-Gruet F, Jeannin A, Dubois-Arber F (2006). Long-term HIV/AIDS-related prevention behaviours among men having sex with men: Switzerland 1992-2000. AIDS Care 18(1): 35-43.

Moreau-Gruet F, Jeannin A, Dubois-Arber F, Spencer B (2001). Management of the risk of HIV infection in male homosexual couples. AIDS 15(8): 1025-35.

Office fédéral de la santé publique (OFSP) (2004). Epidémiologie du VIH en Suisse: depuis 2002 les tests VIH positifs demeurent à un niveau élevé. Bulletin OFSP 49: 916-22.

Ross MW, Tikkanen R, Mansson SA (2000). Differences between Internet samples and conventional samples of men who have sex with men: implications for research and HIV interventions. Soc Sci Med 51(5): 749-58.

Schiltz MA (1997). Parcours de jeunes homosexuels dans le contexte du VIH: la conquête de modes de vie. Population 52(6): 1485-538. 
Stolte IG, Dukers NH, Geskus RB, Coutinho $R A$, de Wit JB (2004). Homosexual men change to risky sex when perceiving less threat of HIV/AIDS since availability of highly active antiretroviral therapy: a longitudinal study. AIDS 18(2): 303-9.

Van de Ven P, Rawstorne P, Nakamura T, Crawford J, Kippax $S$ (2002). HIV treatments optimism is associated with unprotected anal intercourse with regular and with casual partners among Australian gay and homosexually active men. Int J STD AIDS 13(3): 181-3.
Van de Ven P, Rawstorne P, Treloar C, Richters $J$. (2004). HIV/AIDS, hepatitis and sexually transmissible infections in Australia: annual report of behaviour. Sydney: National Centre in HIV Social Research.

Williamson LM, Hart GJ (2004). HIV optimism does not explain increases in high-risk sexual behaviour among gay men in Scotland. AIDS 18(5): 834-5.

\section{Adresse pour correspondence}

Hugues Balthasar

Institut universitaire de médecine sociale

et préventive (IUMSP)

Unité d'évaluation de programmes de

prévention (UEPP)

Rue du Bugnon 17

CH-1005 Lausanne

Courriel: Hugues.Balthasar@chuv.ch
To access this journal online: http://www.birkhauser.ch/IJPH 